Case Report

\title{
A Patient with a Right Atrium Mass and Congenital Heart Disease: A Challenging Diagnosis of a Stubborn Disease
}

\author{
Wenyan Wang, ${ }^{1}$ Huaicong Long, ${ }^{2}$ and Zhiying Zhao ${ }^{2}$ \\ ${ }^{1}$ Heart Failure Center, Sichuan Provincial People's Hospital, Chengdu, Sichuan 610072, China \\ ${ }^{2}$ Geriatric ICU, Sichuan Provincial People's Hospital, Chengdu, Sichuan 610072, China \\ Correspondence should be addressed to Wenyan Wang; ddmm611@163.com
}

Received 18 February 2016; Revised 12 July 2016; Accepted 1 August 2016

Academic Editor: Assad Movahed

Copyright (C) 2016 Wenyan Wang et al. This is an open access article distributed under the Creative Commons Attribution License, which permits unrestricted use, distribution, and reproduction in any medium, provided the original work is properly cited.

\begin{abstract}
Cardiac lymphoma is extremely rare. An intracardiac mass has rarely been reported to be the cardiac involvement of extranodal lymphoma. It is difficult to establish a final diagnosis via routine examinations. The ability of an echocardiogram to characterize tissue is limited; systemic (18)F-FDG PET/CT scans provide important information for both staging and response assessment in patients with lymphoma. A 68-year-old Chinese male with a second patent foramen ovale (PFO) and an interventricular septal defect presented at our institute with persistent fever, shortness of breath, repeated paroxysmal supraventricular tachycardia (PSVT) attack, and rapidly progressing superior vena cava syndrome. The patient also presented with a mass located in the upper right atrium and superior vena cava which was detected by echocardiogram. (18)F-FDG PET/CT scan revealed a pathological increase of (18)F-FDG uptake in the atrial mass and several other extracardiac lymph nodes. Lymph node biopsy was positive for large B-cell lymphoma. Immunohistochemistry revealed intense and diffuse expression of CD20, CD10, BCL-6, and Ki-67. The patient died without any chemotherapy 18 days after hospital discharge.
\end{abstract}

\section{Introduction}

Cardiac masses arising from the heart or the pericardium are rare [1] and potentially lethal. Cardiac Non-Hodgkin's lymphoma (NHL) is extremely rare and accounts for $1-2 \%$ of primary cardiac tumors involving the right versus left atrium at a ratio of approximately $8: 1[2,3]$. Older patients with untreated congenital heart disease and the comorbidity of cardiac lymphoma are even less common and have a poor prognosis. We report the case of an old man who had initially presented with $\mathrm{PFO} / \mathrm{VSD}$ heart failure and endocarditis and then was later diagnosed with diffuse large B-cell lymphoma (DLBCL) in his right atrium after a mass was discovered via $\mathrm{PET} / \mathrm{CT}$.

\section{Case Report}

A 68-year-old Chinese male presented to the geriatric ICU in May, 2015, with a "persistent fever lasting two months and worsening shortness of breath lasting one month." Two months prior to presenting to the ICU, the patient began to experience shivering and hyperpyrexia reaching $40^{\circ} \mathrm{C}$ and a cough with phlegm production. He denied chest pain and hemoptysis. One month prior to presenting to the ICU, he began to complain of palpitations and edema in his lower extremities. He received antibiotics and diuretics without any progress. Although he was previously diagnosed with chronic bronchitis and pulmonary emphysema many years earlier, PFO and VSD remained untreated. He has been a smoker for 40 years and there is nothing significant in his family history.

Vital signs at time of admission were as follows: temperature $38.8^{\circ} \mathrm{C}$, pulse $150 \mathrm{bpm}$, being conscious and anicteric, no cyanosis, and an operative lymph node palpable in the left iliac region without tenderness. Coarse crackles were heard at the base of lungs bilaterally, as well as cardiac murmurs at the third and fourth intercostal space on the left bounder of the sternum. Moderate pitting edema of both lower extremities was also observed. Laboratory tests were WBC $7.37 \times 10 \mathrm{E} 9 / \mathrm{l}$, $\mathrm{N} 75.8 \%$, Hb $105 \mathrm{~g} / \mathrm{L}$, C-reactive protein (CRP) $89.15 \mathrm{mg} / \mathrm{L}$, BNP $523 \mathrm{pg} / \mathrm{mL}$, PCT 0.25 , and ESR $95 \mathrm{~mm} / \mathrm{h}$. Renal function and liver enzymes were within normal limits. Blood plasmodium test and ANA spectrum were negative. Blood culture was 




FIGURE 1: Upper right atrium and superior vena cava mass. Subcostal view of dual atrium demonstrated a lobulated and echo reflectant mass (arrow) in upper right atrium and superior vena cava.

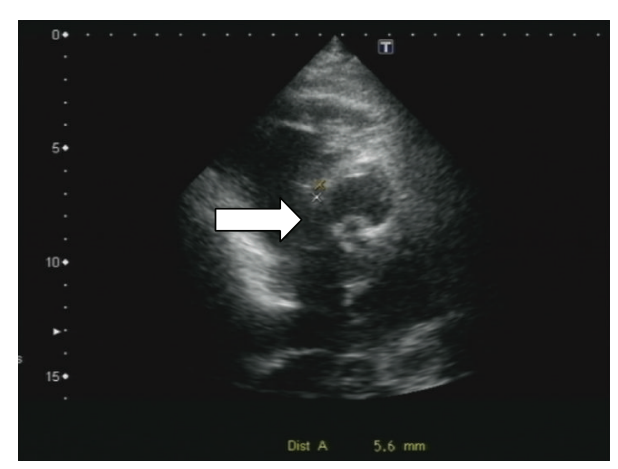

Figure 2: Ventricular septal defect (VSD). Parasternal short view showed the VSD (arrow).

Staphylococcus hominis (sensitive to vancomycin). ECG was paroxysmal supraventricular tachycardia (PSVT) with HR at 155 beats/min. Chest X-ray showed there was some patchy consolidation at the right upper lung and bilateral pleural effusion. The lymph nodes with reactive hyperplasia were detected by ultrasound at bilateral inguinal regions.

Transthoracic echocardiography (TTE) is shown in Figures 1-2.

After his admission, the patient was put on intravenous cefoperazone-tazobactam $2.5 \mathrm{~g}$ q12h for 16 days and intravenous Vancomycin 500 thousand $\mathrm{q} 8 \mathrm{~h}$ for 5 days and then increased to q6h for 11 days. The patient was also put on Propafenone, metoprolol, Perindopril, diuretics, and glyceryl trinitrate. He continued to experience worsening shortness of breath and orthopnea and swelling in his right upper limb, and ECG telemonitoring showed repeated attack of PSVT. The patient was suspected to have developed superior vena cava syndrome; furthermore, the patient received a vascular ultrasound with no thrombosis detected, and BNP reduced to $159 \mathrm{ng} / \mathrm{mL}$. Systemic (18)F-FDG PET/CT scan (Figures 3(a) and 3(b)) revealed that the (18)F-FDG uptake in the mass located in the right atria pathologically increased. The patient finally received lymph node biopsy: large B-cell lymphoma, CD20(+) CD3 T cell(+) Bcl-6(+), CK(+) CD21(-) CD10(-) MUM-1(-) EBER(-). The patient and his family declined chemotherapy and he was discharged from the hospital. He died after 18 days of following up.

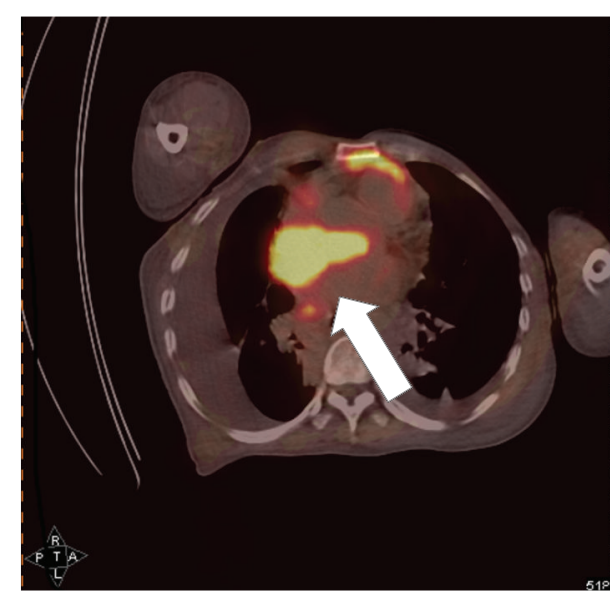

(a)

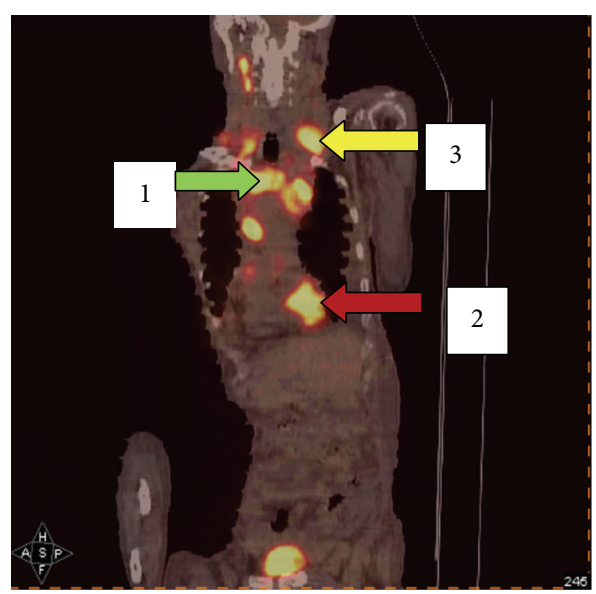

(b)

FIGURE 3: Systemic (18)F-FDG PET/CT scan revealed that the (18)FFDG uptake in the mass located in the right atria pathologically increased ((a) arrow). Quite a few lymph nodes with increased uptake of (18)F-FDG were revealed at the regions of neck (yellow arrow), mediastinum (green arrow), and right atrium (red arrow) (b).

\section{Discussion}

The incidence of NHL has increased in recent decades in Asia [4]. But lymphomas rarely involve the heart with incidence between $9 \%$ and $24 \%$ on autopsy series. NHL can affect the heart but usually in the form of pericardial effusion which is often associated with direct epicardial spread [5]. Only 0.5\% of all extranodal lymphomas present as cardiac neoplasms [6].

Cardiac masses arising in the heart or the pericardium are potentially lethal; they have variable symptoms which lack specificity; the clinical presentation is determined utilizing numerous factors. Depending on tumor location, size, growth rate, degree of invasion, and friability, the clinical presentations could include fever, fatigue, arthralgia, refractory congestive heart failure or arrhythmia, cardiomegaly, pericardial effusion, sudden death [7], and superior vena cava syndrome. The rarity and heterogeneous clinical presentation of PCL 
make its diagnosis difficult. Imaging studies such as TEE may not be reliable in distinguishing cardiac lymphoma from other cardiac neoplasms including myxomas, angiosarcoma, or rhabdomyomas. Endomyocardial biopsy, excisional intraoperative biopsy, and pericardial fluid cytological evaluation should be utilized to establish final diagnosis.

We are reporting this case of NHL and massive intracardiac involvement diagnosed by echocardiographic and PET/CT assessment. The patient's heart defect, medical history of lung disease, persistent fever, and positive blood culture are what guided the original misdiagnosis of hematosepsis and endocarditis. It is hard to explain how come even BNP decreased but refractory arrhythmia and superior vena cava syndrome were getting worsen based on aggressive treatment of antibiotics, diuretics, and vassal dilator. Although TTE is the preferred initial imaging technique used in the detection and diagnosis of cardiac masses, its ability to characterize tissue is limited. (18)F-FDG PET/CT scanning is superior in this regard and, due to its noninvasive approach, should therefore be the diagnostic tool of choice for cardiac mass assessment for the elderly, particularly those who might lose the chance to receive invasive procedures such as TEE or myocardial biopsy. The sensitivity of FDG PET/CT imaging was reported to be $71-100 \%$, with a specificity of $69-100 \%$ and a negative predictive value of $80-100 \%[8,9]$.

The prognosis for patients with cardiac sarcomas remains very poor [10]. DLBCL is an aggressive lymphoma that is typically treated with chemotherapy and radiation therapy which could improve the survival rates [11]. Early systemic chemotherapy, in addition to restricting the tumor, might yield a better prognosis.

\section{Competing Interests}

The authors declare that they have no competing interests.

\section{References}

[1] K. Y. Lam, P. Dickens, and A. C. L. Chan, "Tumors of the heart: a 20-year experience with a review of 12485 consecutive autopsies," Archives of Pathology and Laboratory Medicine, vol. 117, no. 10, pp. 1027-1031, 1993.

[2] G. L. Ceresoli, A. J. Ferreri, E. Bucci, C. Ripa, M. Ponzoni, and E. Villa, "Primary cardiac lymphoma in immunocompetent patients: diagnostic and therapeutic management," Cancer, vol. 80, pp. 1497-1506, 1997.

[3] B. Delmas-Marsalet, V. Molinie, L. Jary et al., "Cardiac localization of non-Hodgkin's lymphoma: two case reports and review of the literature," Nouvelle Revue Francaise d'Hematologie, vol. 37, no. 4, pp. 223-230, 1995.

[4] J. Ferlay, H.-R. Shin, F. Bray, D. Forman, C. Mathers, and D. M. Parkin, "Estimates of worldwide burden of cancer in 2008: GLOBOCAN 2008," International Journal of Cancer, vol. 127, no. 12, pp. 2893-2917, 2010.

[5] P. J. McDonnell, R. B. Mann, and B. H. Bulkley, "Involvement of the heart by malignant lymphoma: A Clinicopathologic Study," Cancer, vol. 49, no. 5, pp. 944-951, 1982.

[6] C. E. Miguel and R. B. Bestetti, "Primary cardiac lymphoma," International Journal of Cardiology, vol. 149, no. 3, pp. 358-363, 2011.
[7] P. Cairns, J. Butany, J. Fulop, H. Rakowski, and S. Hassaram, "Cardiac presentation of non-Hodgkin's lymphoma," Archives of Pathology and Laboratory Medicine, vol. 111, no. 1, pp. 80-83, 1987.

[8] S. A. Johnson, A. Kumar, M. J. Matasar, H. Schöder, and J. Rademaker, "Imaging for staging and response assessment in lymphoma," Radiology, vol. 276, no. 2, pp. 323-338, 2015.

[9] S. N. Reske, "PET and restaging of malignant lymphoma including residual masses and relapse," European Journal of Nuclear Medicine and Molecular Imaging, vol. 30, supplement 1, pp. S89-S96, 2003.

[10] L. Simpson, S. K. Kumar, S. H. Okuno et al., "Malignant primary cardiac tumors: review of a single institution experience," Cancer, vol. 112, no. 11, pp. 2440-2446, 2008.

[11] J. K. C. Chan, K. Aozasa, and P. Gaulard, "Diffuse large Bcell lymphoma associated with chronic inflammation," in World Health Organization Classification of Tumours: Pathology and Genetics of Tumours of Haematopoietic and Lymphoid Tissues, S. H. Swerdlow, E. Campo, and N. L. Harris, Eds., pp. 245-246, IARC Press, Lyon, France, 4th edition, 2008. 


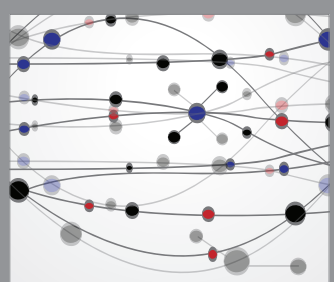

The Scientific World Journal
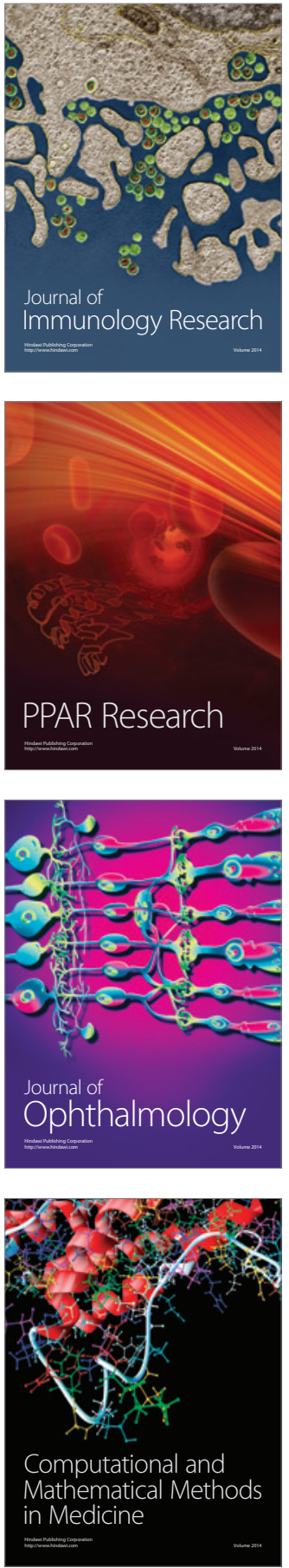

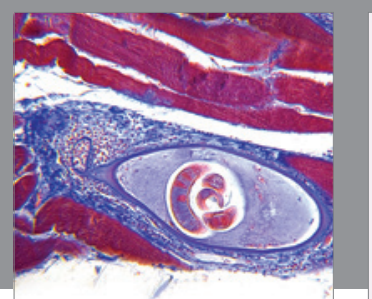

Gastroenterology Research and Practice

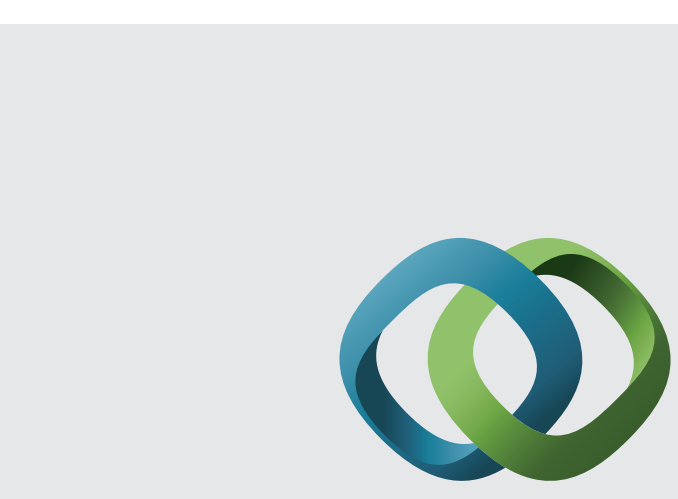

\section{Hindawi}

Submit your manuscripts at

http://www.hindawi.com
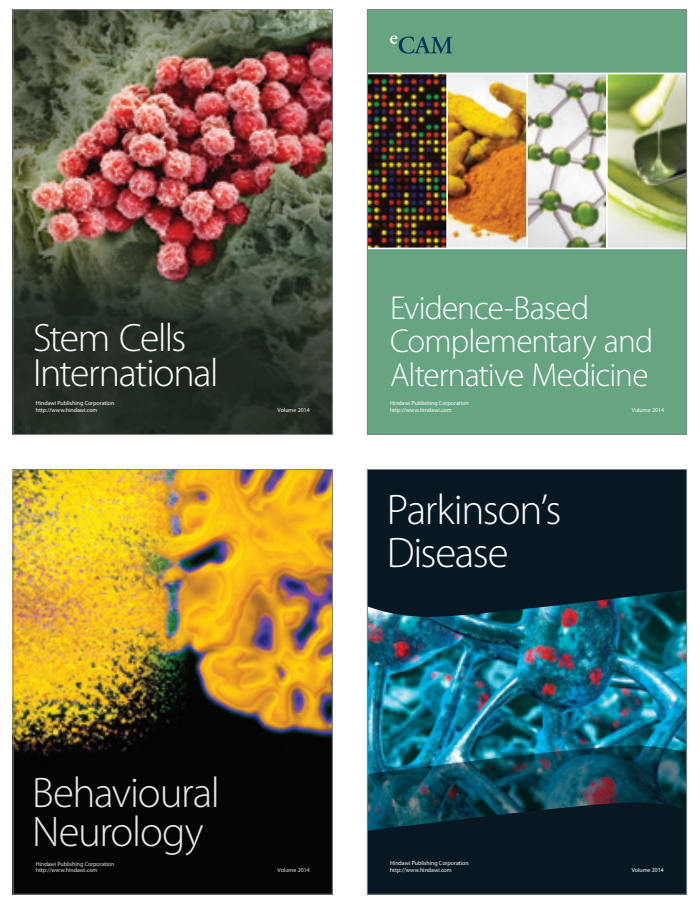
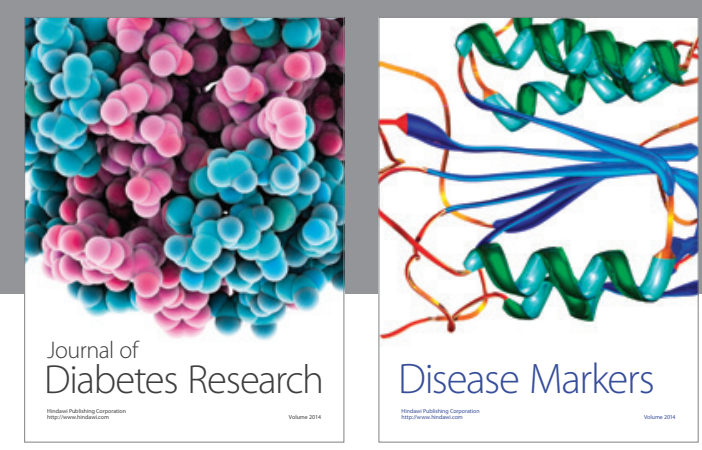

Disease Markers
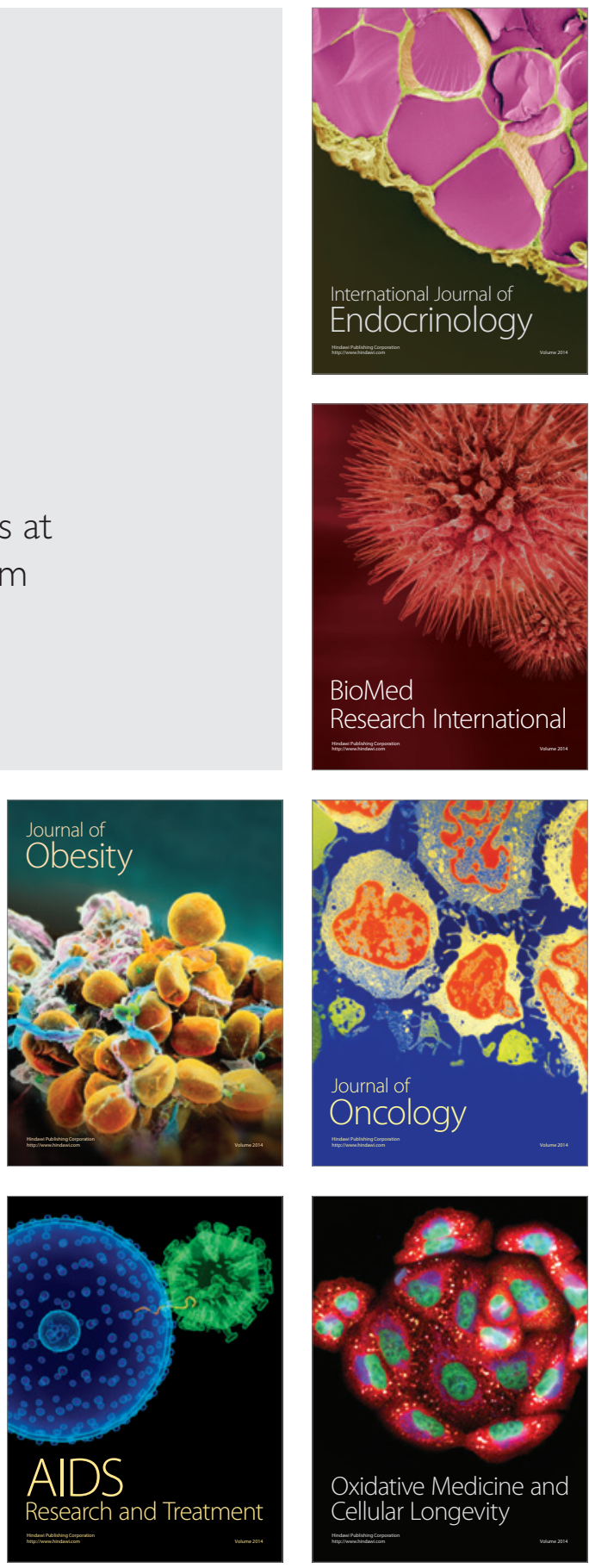